\title{
CIS is a negative regulator of IL-15-mediated signals in NK cells
}

\author{
Cristina Bottino $^{1,2}$, Alessandra Dondero ${ }^{1}$, Alessandro Moretta ${ }^{1,3}$, Roberta Castriconi $^{1,3}$ \\ ${ }^{1}$ Dipartimento di Medicina Sperimentale, Università degli Studi di Genova, Genoa, Italy; ${ }^{2}$ Istituto Giannina Gaslini, U.O.C. Immunologia Clinica \\ e Sperimentale, Genoa, Italy; ${ }^{3}$ Centro di Eccellenza per la Ricerca Biomedica (CEBR), U.O. Immunologia Molecolare, Università degli Studi di \\ Genova, Genoa, Italy \\ Correspondence to: Roberta Castriconi. Dipartimento di Medicina Sperimentale, Università degli Studi di Genova, Via G.B. Marsano 10, 16132 \\ Genoa, Italy; Centro di Eccellenza per la Ricerca Biomedica (CEBR), U.O. Immunologia Molecolare, Università degli Studi di Genova, Via de Toni \\ 14, 16132 Genoa, Italy. Email: roberta.castriconi@unige.it.
}

Submitted Sep 20, 2016. Accepted for publication Sep 23, 2016.

doi: 10.21037/tcr.2016.10.79

View this article at: http://dx.doi.org/10.21037/tcr.2016.10.79

The cytokine-inducible SH2-containing protein (CIS, encoded by Cish) is a member of the suppressors of cytokine signaling (SOCS), a family of intracellular proteins that have been show to regulate the activity of a variety of cytokine and growth factor receptors (1). Similar to the other members, CIS presents a central SH2-domain able to interact with phosphotyrosine residues and a SOX box motif that recruits the ubiquitin-transferase system (u-ts) and directs targeted proteins to proteasomal degradation.

CIS has also been shown to interact with the cytoplasmic domains and suppress the signals of different receptors including the IL-2R (2). In the study recently published in nature immunology (3), Delconte and colleagues analyze the role of CIS in regulating the IL-15 signaling pathway in NK cells. This pathway includes signals that are transmitted through the IL-2R $\beta$ (CD122) and $\gamma c$ (CD132) subunits that, upon engagement, associate and activate JAK1 and JAK3 (Figure 1). It follows STAT5 activation that induces the transcription of genes involved in the survival, proliferation and function of NK cells (4). The authors deepened the regulatory role of CIS in mouse NK cells using a Cish-deleted model $\left(\right.$ Cish $\left.^{-1-}\right)$. They show that CIS is expressed early during NK cells differentiation and that saturating high concentrations of IL-15 increased its expression at both mRNA and protein level. Cish $^{-/-}$NK cells showed upregulation of IL-2R $\beta$ expression and hyper-responsiveness to IL-15, which resulted in increased survival, proliferation, cytotoxicity and IFN- $\gamma$ production as compared to $\mathrm{Cish}^{+/+} \mathrm{NK}$ cells (Figure 1). Moreover, in Cish ${ }^{-/}$NK cells cytokine stimulation modulated more than 1,000 genes, including those coding for granzymes, and increased phosphorylation of 69 kinases including JAK-1, JAK-3, STAT5 and kinases mainly involved in regulating cellular proliferation such as CDK1/2, Prkr and Aurora kinases. By using a recombinant trimeric complex formed by a human CIS-SH2 construct coupled to elongins B and C, the authors show that CIS, similar to SOCS-1 and SOCS-3, binds to JAK-1 and JAK3. CIS, which lacks the kinase inhibitor region (KIR), interact with JAK1 via the SH2 domain, targets JAK1 to proteosomal degradation and inhibits its enzymatic activity (Figure 1), although with an efficacy 100 fold lower than SOCS1. It has been previously published that exposure of mouse NK cells to high concentrations of IL-15 results in the activation of the evolutionarily conserved serinethreonine kinase mTOR (mammalian target of rapamycin complex) a crucial metabolic checkpoint regulating translation and glycolytic pathway in both homeostatic and pro-inflammatory conditions (5). Although CIS and mTOR share analogy in the mechanisms of induction, the two pathways appear to be disconnected. Indeed, in $\mathrm{Cish}^{-1-} \mathrm{NK}$ cells IL-15 does not increase the phosphorylation of AKT, that represents a crucial event during mTOR activation (6). In line with this observation, Cish $^{-1-} \mathrm{NK}$ cells show normal mitochondrial respiration and glycolysis, functions that are regulated by the AKT activity.

$\mathrm{Cish}^{-/-}$mice apparently are healthy and display normal NK and T lymphocytes frequency and phenotype (3). According to an increased activity of CIS-deficient immune cells, Cish ${ }^{-/-}$mice show fewer lung metastases than $\mathrm{Cish}^{+++}$ when intravenous injected with melanoma, prostate or breast cancer tumor cell lines, a protective effect that is 


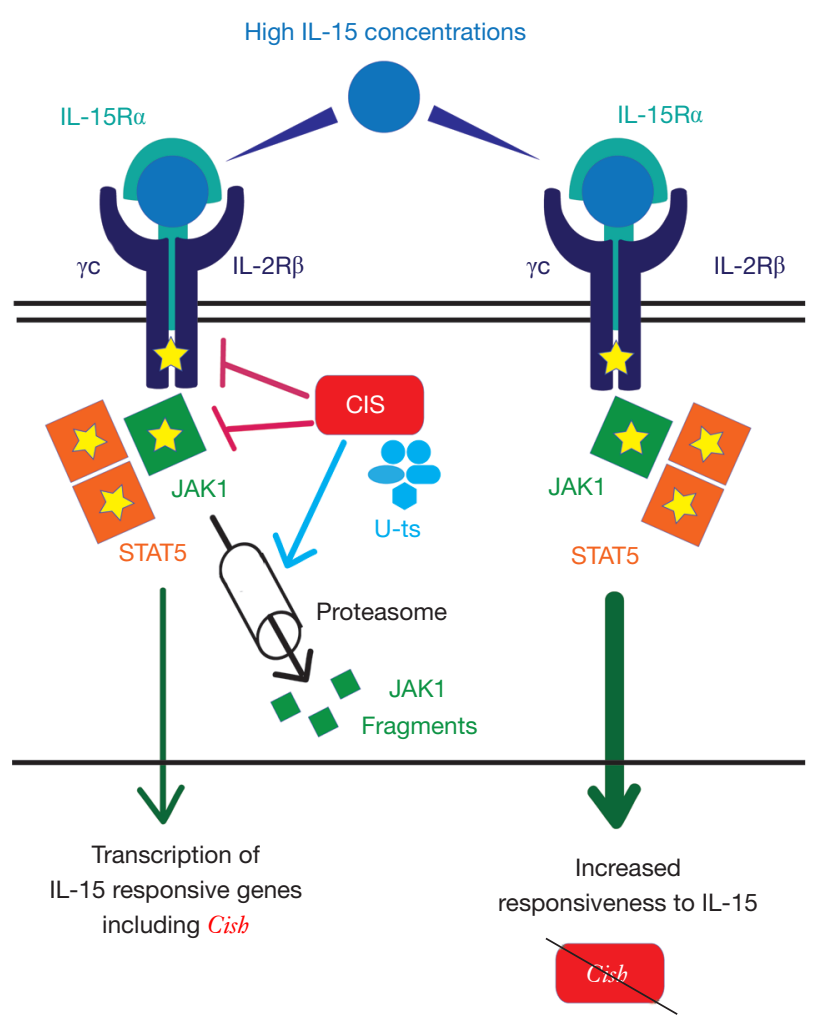

Figure 1 Regulatory role of CIS in IL-15 stimulated NK cells. The IL-15 binding to the receptor activates JAK1 (JAK3) and STAT5 leading to the transcription of genes regulating survival, proliferation and effector functions of NK cells. A high IL-15 concentration leads to the transcription of Cish whose product binds IL-2R $\beta$ and JAKs. CIS inhibits JAK1 tyrosine phosphorylation and drives JAK1 to proteasomal degradation thanks to the recruitment of the ubiquitin-transferase system (u-ts). In $\mathrm{Cish}^{-1-}$ mice NK cells show increased responsiveness to IL-15. CIS, cytokine-inducible SH2-containing protein.

mainly dependent on the effector function of NK cells. Finally, even fewer metastases were detected in $\mathrm{Cish}^{-1-}$ mice treated with a combination of anti-PD-1 and antiCTLA-4 mAbs (7-9). Since CIS is induced by high concentrations of IL-15, the authors suggest that, in the mouse model used, NK cells might receive such intense stimulus from the inflammatory tumor microenvironment. In particular, immune cells colonizing tumor tissues, such as dendritic cells or macrophages, might "trans-present" high concentrations of IL-15 to NK cells.

Overall, the study demonstrates that CIS represents a negative feedback mechanism activated not only by IL-2 (2) but also by IL-15 switching off NK cells effector functions including cytotoxicity against tumor cells $(4,10,11)$. In this context, a recent paper showed that in CD8+ T cells CIS inhibits the TCR signaling by physically interacting with the TCR intermediate PLC- $\gamma 1$ and targeting it for proteasomal degradation (12). The Authors also propose CIS as a novel immune checkpoint and highlight the potential benefit of therapies combining CIS inhibition and low doses of IL-15 to improve anti-tumor NK cell function in patients.

Importantly however, the information on CIS is still scarce and we cannot underestimate the possible side effects of unleashing the function of lymphocytes with high cytolytic and proliferative capacity. Moreover, a conditio sine qua non will be to inactivate CIS in selected and specific target cells. It can't be disregarded previous observations showing that CIS expression is not restricted to lymphocytes but is present in other normal tissues including kidney, lung, liver, stomach and heart thus suggesting that it may represent a wide type of regulatory molecule $(1,13)$. Moreover, CIS was shown to interact and regulate highly pleiotropic receptors including the growth hormone (1). Although $\mathrm{Cish}^{-/-}$mice were healthy, fertile and with no evidence of phenotypic or immunological abnormalities, further studies are needed to exclude that CIS targeting might result in dangerous dysregulation or breaking of crucial feedback mechanisms that tune important cellular functions in both hematopoietic and non-hematopoietic tissues. At this regard, a recent study shows that aged $\mathrm{Cish}^{-1-}$ mice develop an inflammatory lung condition associated with altered IL-4-STAT6 and IL-2-STAT5 signaling in CD4+ T cells (14). Moreover CIS and SOCS1-7 would play a key role in regulation of cellular homeostasis and inflammation and are now regarded as tumor suppressors both in hematological and solid malignances (1). Indeed, a link has been demonstrated between reduced levels/ function of SOCS proteins (due to gene mutation/deletion or epigenetic mechanisms) and cancer development and progression (1). The role of some proteins belonging to this family could be even more complex. Indeed, SOCS-2, which is closely related to CIS (1), appears to play a dual opposite role, being able to control both growth factor receptors and other SOCS proteins depending on its concentration and cellular context. Last but not least, it is important to note that an untargeted CIS neutralization might strength not only the immune-stimulatory effects of IL-15 but also its pro-angiogenic property (15-17).

In conclusion, the results by Delconte (and other research groups) contribute to our knowledge on CIS 
properties. However, data must be validated in the human system and further studies are required to demonstrate the absence of long-term systemic side effects of CIS inactivation and to translate the results into the clinical practice.

\section{Acknowledgements}

Funding: This work was supported by the Associazione Italiana per la Ricerca sul Cancro (AIRC): Investigator Grant (No. 15704) to A Moretta and Special Program Molecular Clinical Oncology 5 per 1000 (No. 9962) to A Moretta.

\section{Footnote}

Provenance: This is a Guest Commentary commissioned by Section Editor Qian Chen (Center for Inflammation \& Epigenetics, Houston Methodist Hospital Research Institute, Houston, TX, USA).

Conflicts of Interest: A Moretta is a founder and shareholder of Innate-Pharma (Marseille, France). The other authors have no conflicts of interest to declare.

Comment on: Delconte RB, Kolesnik TB, Dagley LF, et al. CIS is a potent checkpoint in NK cell-mediated tumor immunity. Nat Immunol 2016;17:816-24.

\section{References}

1. Sasi W, Sharma AK, Mokbel K. The role of suppressors of cytokine signalling in human neoplasms. Mol Biol Int 2014;2014:630797.

2. Aman MJ, Migone TS, Sasaki A, et al. CIS associates with the interleukin-2 receptor beta chain and inhibits interleukin-2-dependent signaling. J Biol Chem 1999;274:30266-72.

3. Delconte RB, Kolesnik TB, Dagley LF, et al. CIS is a potent checkpoint in NK cell-mediated tumor immunity. Nat Immunol 2016;17:816-24.

4. Huntington ND. The unconventional expression of IL15 and its role in NK cell homeostasis. Immunol Cell Biol 2014;92:210-3.

5. Marçais A, Cherfils-Vicini J, Viant C, et al. The metabolic checkpoint kinase mTOR is essential for IL-15 signaling during the development and activation of NK cells. Nat Immunol 2014;15:749-57.
6. Laplante M, Sabatini DM. mTOR signaling in growth control and disease. Cell 2012;149:274-93.

7. Pardoll DM. The blockade of immune checkpoints in cancer immunotherapy. Nat Rev Cancer 2012;12:252-64.

8. Shin DS, Ribas A. The evolution of checkpoint blockade as a cancer therapy: what's here, what's next? Curr Opin Immunol 2015;33:23-35.

9. Pitt JM, Vétizou M, Daillère R, et al. Resistance Mechanisms to Immune-Checkpoint Blockade in Cancer: Tumor-Intrinsic and -Extrinsic Factors. Immunity 2016;44:1255-69.

10. Verdeil G, Puthier D, Nguyen C, et al. STAT5-mediated signals sustain a TCR-initiated gene expression program toward differentiation of CD8 T cell effectors. J Immunol 2006;176:4834-42.

11. Huntington ND, Puthalakath H, Gunn P, et al. Interleukin 15 -mediated survival of natural killer cells is determined by interactions among Bim, Noxa and Mcl-1. Nat Immunol 2007;8:856-63.

12. Palmer DC, Guittard GC, Franco Z, et al. Cish actively silences TCR signaling in CD8+ T cells to maintain tumor tolerance. J Exp Med 2015;212:2095-113.

13. Yoshimura A, Ohkubo T, Kiguchi T, et al. A novel cytokine-inducible gene CIS encodes an SH2-containing protein that binds to tyrosine-phosphorylated interleukin 3 and erythropoietin receptors. EMBO J 1995;14:2816-26.

14. Yang XO, Zhang H, Kim BS, et al. The signaling suppressor CIS controls proallergic T cell development and allergic airway inflammation. Nat Immunol 2013;14:732-40.

15. Angiolillo AL, Kanegane H, Sgadari C, et al. Interleukin-15 promotes angiogenesis in vivo. Biochem Biophys Res Commun 1997;233:231-7.

16. Kuniyasu H, Ohmori H, Sasaki T, et al. Production of interleukin 15 by human colon cancer cells is associated with induction of mucosal hyperplasia, angiogenesis, and metastasis. Clin Cancer Res 2003;9:4802-10.

17. Villadsen LS, Schuurman J, Beurskens F, et al. Resolution of psoriasis upon blockade of IL-15 biological activity in a xenograft mouse model. J Clin Invest 2003;112:1571-80.

Cite this article as: Bottino C, Dondero A, Moretta A, Castriconi R. CIS is a negative regulator of IL-15-mediated signals in NK cells. Transl Cancer Res 2016;5(Suppl 4):S875S877. doi: 10.21037/tcr.2016.10.79 